ИЗГИБНЫЕ КОЛЕБАНИЯ КОРОНАЛЬНЫХ ПЕТЕЛЬ, СОДЕРЖАЩИХ ЭЛЕКТРИЧЕСКИЕ ТОКИ

\author{
Михаляев Б.Б., Дертеев С.Б., Шивидов Н.К., Тухкин И.Ю. \\ Калмыикий государственный университет, Элиста, Россия
}

\title{
KINK OSCILLATIONS OF CORONAL LOOPS WITH ELECTRIC CURRENTS
}

\author{
Mikhalyaev B.B., Derteev S.B., Shividov N.K., Tukhkin I.Yu. \\ Kalmyk State University, Elista, Russia
}

Kink oscillations on coronal loops is one of main objects of the study in the coronal seismology, they are observed usually in the extreme ultraviolet emission. Its properties are studied well in the approximation of the linear magnetohydrodynamics for a model of a uniform magnetic tube. Now we study the kink-mode of coronal loops that consists of a cylindrical core with axial magnetic field and coaxial annulus with purely azimuthal magnetic field. One can expect that the formation of such magnetic flux tubes consisting of two parts is a natural result of the evolution of twisted magnetic tubes transported in the solar atmosphere from the convection zone. New wave solutions are presented in the annulus that have singularity in a nonzero point. A solution of the dispersion equation for kink-mode shows undamping free kink oscillations.

\section{DOI: 10.31725/0552-5829-2019-297-300}

Изгибные колебания корональных петель, непосредственно наблюдаемые в крайнем ультрафиолетовом диапазоне электромагнитных волн, являются одним из основных объектов исследования в корональной сейсмологии [1-3]. МГД-теория линейных колебаний корональных магнитных трубок подробно разработана для случая модели однородной магнитной трубки в целом ряде работ [4-5]. В настоящей работе рассматриваются колебания магнитной трубки, содержащей продольную компоненту электрического тока. Подобная трубка может служить моделью корональной петли, в которой электрический ток течет вдоль петли от одного основания к другому. Концепция продольного электрического тока лежит в основе модели простых петельных вспышек Альфвена-Карлквиста [2-3].

Мы рассматриваем магнитную трубку, состоящую из двух коаксиальных, вложенных друг в друга частей [6-7]. Центральная часть, именуемая шнуром, содержит однородное продольное поле и характеризуется альвеновской скоростью $V_{\mathrm{i}}$. Во внешней среде структура поля аналогичная, и альвеновская скорость равна $V_{\mathrm{e}}$. Во внешней части трубки, которую мы называем оболочкой, поле азимутальное и потенциальное: $\left(0, B_{0} / \alpha r, 0\right)$. Здесь $\alpha$ есть постоянный параметр, $r$ - радиальная координата в цилиндрической системе координат, ось $Z$ которой совпадает с осью трубки. Плотность плазмы в оболочке убывает с радиусом как $\rho_{0} / \alpha^{2} r^{2}$ (рис. 1), 
поэтому здесь альвеновская скорость $V_{0}$ постоянна. Ранее нами были изучены радиальные моды подобной трубки [7-8], представляющие собой захваченные осесимметричные быстрые магнитозвуковые волны. В корональных условиях мы пренебрегали газовым давлением по сравнению с магнитным. Из требований равновесия вытекают следующие соотношения $B_{\mathrm{i}} \alpha b=B_{0}=B_{\mathrm{e}} \alpha a$.
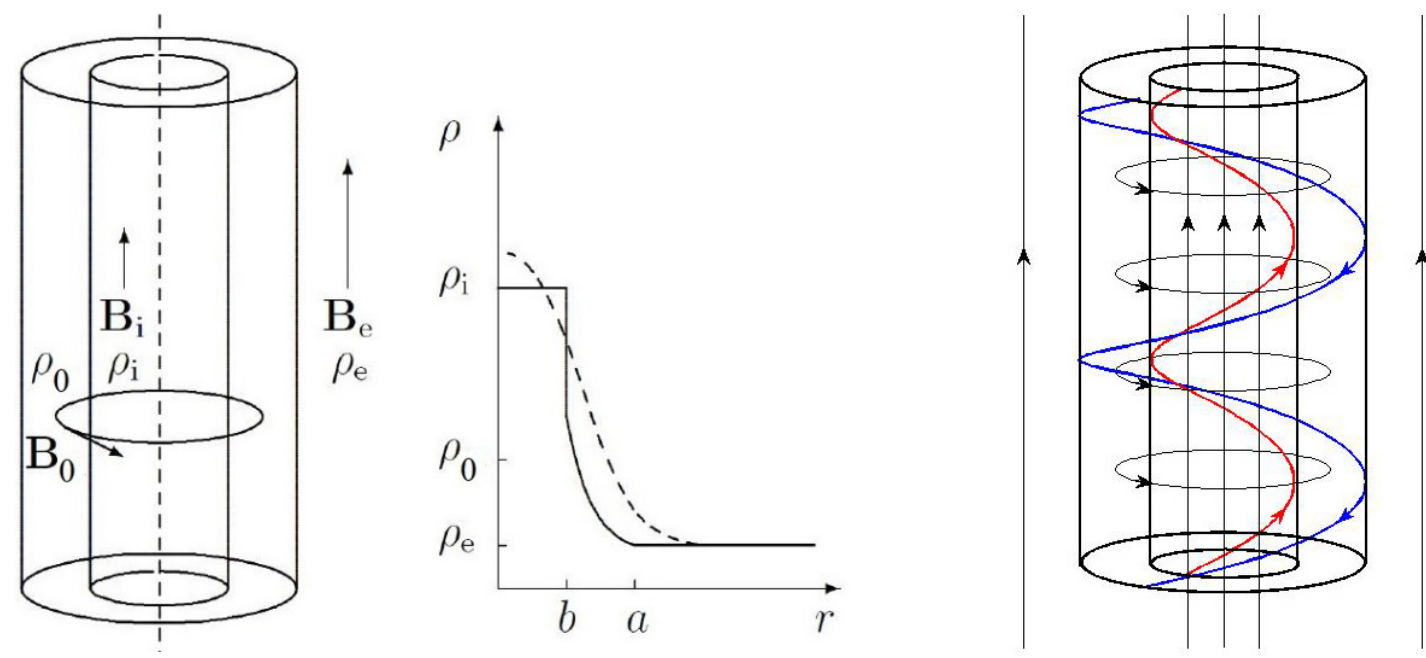

Рис. 1. Общий вид магнитной трубки и распределение плотности плазмы, $b$ есть радиус шнура, $a$ - радиус трубки.

Рис. 2. Распределение электрических токов, имеющих поверхностный характер.

Здесь в тех же условиях мы рассматриваем быстрые магнитозвуковые моды в виде изгибных возмущений. Соответствующее азимутальное число $m=1$. Нас интересует вопрос о существовании незатухающих свободных колебаниях, спектр которых так подробно изучен в случае однородной магнитной трубки. Возмущения записываются в форме

$$
v_{r}=A V(r) \exp (i k z+i \varphi-i \omega t) .
$$

Для радиальной компоненты скорости и возмущения полного давления записываются граничные условия

$$
\begin{aligned}
V_{\mathrm{i}}(b)=V_{0}(b), V_{\mathrm{e}}(a) & =V_{0}(a), \\
P_{\mathrm{i}}(b)=P_{0}(b)+\frac{B_{0}^{2}}{4 \pi i \omega \alpha^{2} b^{3}} V_{0}(b), P_{\mathrm{e}}(a) & =P_{0}(a)+\frac{B_{0}^{2}}{4 \pi i \omega \alpha^{2} a^{3}} V_{0}(a) .
\end{aligned}
$$

Индексом «0» обозначены величины, относящиеся к оболочке. Если волновые распределения в шнуре и во внешней среде даются формулами, справедливыми для незатухающих свободных изгибных колебаний однородной трубки

$$
P_{\mathrm{i}}=A_{1} J_{1}\left(\lambda_{\mathrm{i}} r\right), V_{\mathrm{i}}=\frac{(-i \omega) \lambda_{\mathrm{i}} A_{1} J_{1}\left(\lambda_{\mathrm{i}} r\right)}{\varrho_{\mathrm{i}}\left(\omega^{2}-V_{\mathrm{i}}^{2} k^{2}\right)}, \lambda_{\mathrm{i}}=\sqrt{\frac{\omega^{2}}{V_{\mathrm{i}}^{2}}-k^{2}},
$$




$$
P_{\mathrm{e}}=A_{4} K_{1}\left(\lambda_{\mathrm{e}} r\right), V_{\mathrm{e}}=\frac{(-i \omega) \lambda_{\mathrm{e}} A_{4} K^{\prime}{ }_{1}\left(\lambda_{\mathrm{e}} r\right)}{\varrho_{\mathrm{e}}\left(\omega^{2}-V_{\mathrm{e}}^{2} k^{2}\right)}, \lambda_{\mathrm{e}}=\sqrt{-\frac{\omega^{2}}{V_{\mathrm{e}}^{2}}+k^{2}},
$$

то в оболочке для радиальной зависимости имеется нестандартное уравнение вида

$$
\begin{gathered}
\xi^{2}\left(\xi^{2}-1\right) \frac{d^{2} P_{0}}{d \xi^{2}}+\xi\left(3 \xi^{2}-5\right) \frac{d P_{0}}{d \xi}+\left[\left(1-\chi^{2}\right) \xi^{4}+\left(\chi^{2}-2\right) \xi^{2}-3\right] P_{0} \\
=0, \xi=\frac{r \omega}{V_{0}}, \chi=\frac{V_{0} k}{\omega}
\end{gathered}
$$

Помимо нулевой особой точки, связанной с геометрией задачи, оно имеет особую точку $\xi=1$, имеющую смысл резонанса: на длине окружности радиуса $r=V_{0} / \omega$ укладывается ровно одна длина волны. Для баллонных мод $m>1$ должно укладываться $m$ длин волн.

Решения радиального уравнения (5) мы будем рассматривать в единичном круге

$$
|\xi|<1
$$

используя разложения в окрестности нулевой точки, которые имеют вид

$$
W_{1}=\xi^{-1} \sum_{n \geq 0} a_{n} \xi^{n}, W_{2}=W_{1} \ln \xi+\xi^{-3} \sum_{n \geq 0} b_{n} \xi^{n} .
$$

Коэффициенты разложений находятся с помощью рекуррентных соотношений. Волновое распределение в оболочке записывается в виде

$$
\begin{gathered}
P_{0}=A_{2} W_{1}\left(\lambda_{0} r\right)+A_{3} W_{2}\left(\lambda_{0} r\right), \lambda_{0}=\omega / V_{0}, \\
\left(\xi^{2}-1\right) \frac{\varrho_{0} \omega^{3}}{(-i \omega) \alpha^{2} V_{0}} V_{r 0}=\xi^{2} \frac{d}{d \xi} \xi^{2} P_{0} .
\end{gathered}
$$

Подставляя найденные волновые распределения в граничные условия и приравнивая нулю определитель получающейся системы уравнений для произвольных коэффициентов $A_{1}, A_{2}, A_{3}, A_{4}$, получаем дисперсионное уравнение, связывающее частоту $\omega$ и продольное волновое число $k$.

Решая дисперсионное уравнение относительно $\omega$ для различных значений $k$, нужно иметь в виду условие отсутствия резонанса (6), которое накладывает ограничение сверху на значения волнового числа. Исходя из этого, мы рассматриваем небольшие его значения $(k a \ll 1)$. Результаты

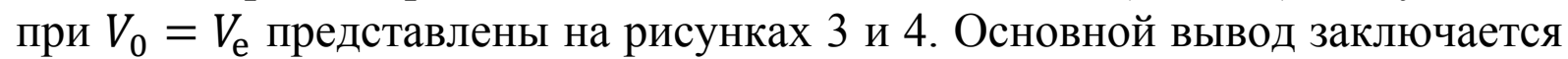
в том, что незатухающие свободные колебания действительно существуют. Более подробный анализ требует рассмотрения других возможных значений $V_{0}$ и изучение роли резонанса в поведении изгибных колебаний. 


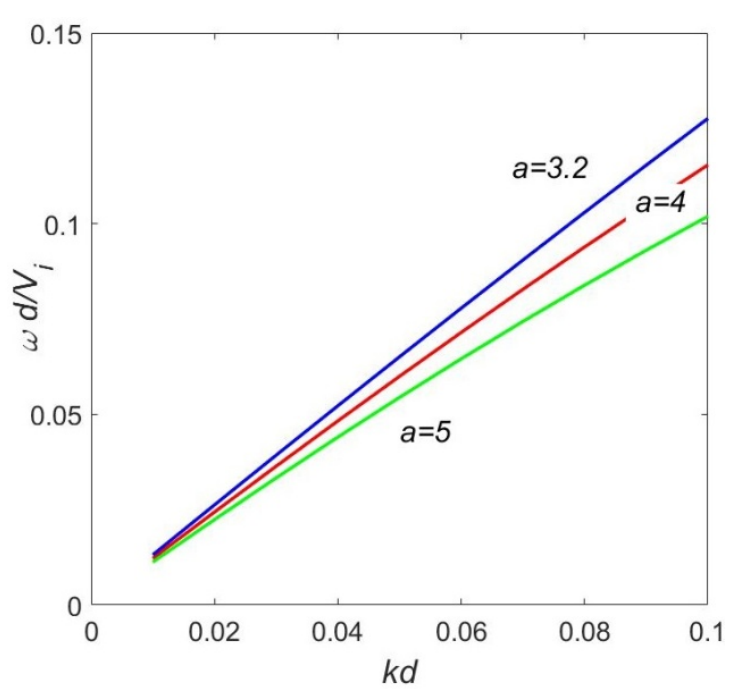

Рис. 3. Кривая зависимости $\omega(k)$.

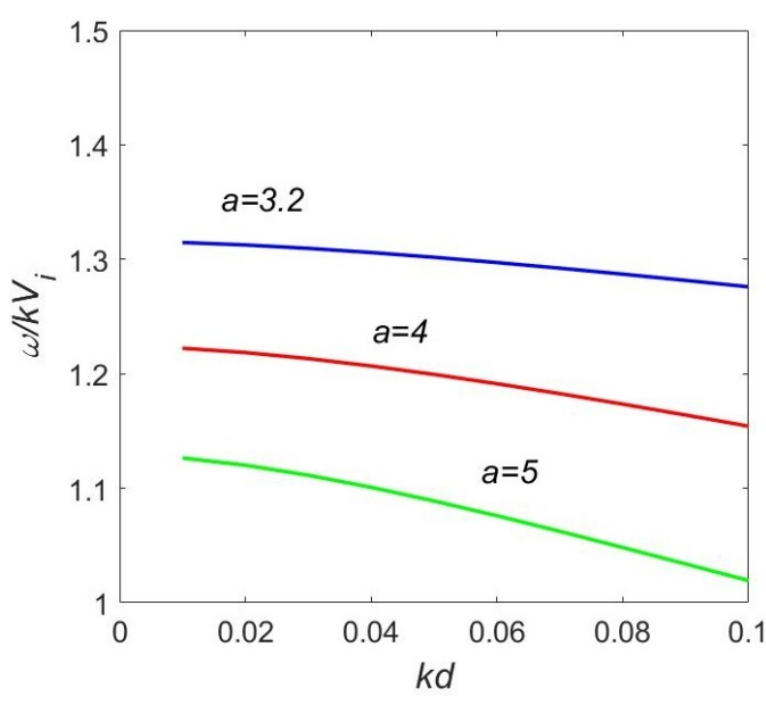

Рис. 4. Кривая зависимости фазовой скорости от волнового числа.

Работа выполнена при поддержке Российского научного фонда (грант № 15-12-20001).

\section{Литература}

1. Aschwanden M.J. Physics of the Solar Corona. An Intoduction with Problems and Solutions. 2005. Springer-Verlag. Berlin, Heidelberg, New York.

2. Зайцев B.B., Степанов А.В. // УФН. 2008. Т. 178. Вып. 11. С. 1165.

3. Stepanov A.V., Zaitsev V.V., Nakariakov V.M. Coronal seismology. 2012. Springer-Verlag. Berlin, Heidelberg, New York.

4. Зайцев В.В., Степанов А.В. // Иссл. геомагн. аэрон. и физике Солнца. 1975. Вып. 37. C. 3 .

5. Edwin P.M., Roberts B. // Solar Phys. 1983. V. 88. P. 179.

6. Михаляев Б.Б. // Письма в Астрон. журнал. 2005. Т. 31. № 6. С. 456.

7. Михаляев Б.Б., Хонгорова О.В. // Письма в Астрон. журнал. 2012. Т. 38. № 10. С. 746.

8. Khongorova O.V., Mikhalyaev B.B., Ruderman M.S. // Solar Phys. 2012. V. 280. P. 153. 